\title{
High-fat diet induces systemic B-cell repertoire changes associated with insulin resistance
}

\author{
TD Pham ${ }^{1,2}$, MHY Chng ${ }^{1,2}$, KM Roskin ${ }^{1}$, KJL Jackson ${ }^{1}$, KD Nguyen ${ }^{1}$, J Glanville ${ }^{1,3}$, J-Y Lee ${ }^{1}$, \\ EG Engleman $^{1,2}$ and SD Boyd ${ }^{1}$
}

The development of obesity-associated insulin resistance is associated with B-lymphocyte accumulation in visceral adipose tissue (VAT) and is prevented by B-cell ablation. To characterize potentially pathogenic B-cell repertoires in this disorder, we performed high-throughput immunoglobulin (lg) sequencing from multiple tissues of mice fed high-fat diet (HFD) and regular diet (RD). HFD significantly changed the biochemical properties of Ig heavy-chain complementaritydetermining region-3 (CDRH3) sequences, selecting for IgA antibodies with shorter and more hydrophobic CDRH3 in multiple tissues. A set of convergent antibodies of highly similar sequences found in the VAT of HFD mice but not RD mice showed significant somatic mutation, suggesting a response shared between mice to a common antigen or antigens. These findings indicate that a simple high-fat dietary intervention has a major impact on mouse B-cell repertoires, particularly in adipose tissues.

\section{INTRODUCTION}

Obesity and the metabolic syndrome are growing problems worldwide, contributing significantly to increased mortality, morbidity, and associated health-care costs. Insulin resistance (IR), which precedes type 2 diabetes, is strongly associated with obesity and is also on the rise worldwide. ${ }^{1}$ The etiologies of IR and type 2 diabetes have been attributed to complex genetic factors as well as environmental exposures, with suspicion falling on soluble factors secreted by adipocytes, as well as metabolic abnormalities in muscle tissue associated with increased intracellular lipid content, among other potential mechanisms. ${ }^{2-5}$ Recently, obesity and IR have been shown to manifest deranged profiles in both innate and adaptive immune responses. ${ }^{1}$ Data from Winer et al. ${ }^{6}$ indicated that the adaptive immune system may play an important role in the development of IR in the context of diet-induced obesity. In these studies, B lymphocytes were implicated in the pathogenesis of IR in C57BL/6 mice fed a high-fat diet (HFD); HFD led to increased numbers of $\mathrm{B}$ cells infiltrating the visceral adipose tissues (VAT), and these B cells influenced VAT-resident macrophage and T-cell effector functions. Most importantly, the development of IR was abrogated by B-cell depletion. ${ }^{6} \mathrm{~B}$-cell-null obese mice that received purified immunoglobulin $\mathrm{G}(\mathrm{IgG})$ collected from IR mice showed decreased responses to glucose tolerance tests, suggesting a role for circulating antibody in the phenotype. However, other immunological roles for B cells, such as the presentation of antigens to T cells, or other effector functions, could also be consistent with the results of this study. Notably, $\mathrm{T}$ cells and macrophages were also required for the development of IR. The potential importance of similar B-cell mechanisms in human IR was supported by the finding that IR human subjects showed autoantibodies to a distinct set of autoantigens as compared with insulin-sensitive subjects.

Given these links between obesity, IR, and B-cell biology in mice and humans, we hypothesized that a HFD and resultant obesity might have a significant impact on B-cell populations and the antibody gene rearrangements they express, potentially selecting for autoreactive $\mathrm{B}$ cells or otherwise altering the $\mathrm{B}$-cell repertoire in affected tissues. To assess the effects of HFD on mouse antibody repertoires, we carried out high-throughput DNA sequencing of 24 million antibody heavy chain gene (IGH) rearrangements from B cells expressing IgM, IgG, and IgA isotypes in the blood, spleen, small intestine, large intestine, liver, peritoneal cavity washing, brown adipose tissue, and VAT of mice fed either regular diet (RD) or HFD for 18 weeks. We report that mice on HFD show systematic alterations of IGH

${ }^{1}$ Department of Pathology, Stanford University, Stanford, California, USA. ${ }^{2}$ Stanford Blood Center, Stanford University, Stanford, California, USA and ${ }^{3}$ Department of Microbiology and Immunology, Stanford University, Stanford, California, USA. Correspondence: SD Boyd (sboyd1@stanford.edu)

Received 24 June 2016; accepted 22 February 2017; published online 19 April 2017. doi:10.1038/mi.2017.25 
rearrangements in their B-cell repertoires in a tissue- and isotype-specific manner. We find that HFD leads to increased proportions of IgA-expressing B cells with short and hydrophobic complementarity-determining region-3 (CDRH3) IGH sequences, and increased proportions of IGH rearrangements lacking somatic mutation. A surprising finding in the analysis is that there are a number of highly stereotyped or "convergent" antibody gene rearrangements expressed by the $\mathrm{B}$ cells in the adipose tissues, peritoneal $\mathrm{B}$ cells, and, to a lesser degree, intestinal tissues across numerous individual mice. These gene rearrangements are rare in other tissues of the body. The convergent antibody gene rearrangements in the adipose tissues of HFD mice are notable for having higher somatic hypermutation frequencies, suggesting that they may have undergone affinity maturation in an antigen-driven process. Taken together, these results demonstrate that dietary alterations can cause significant changes in the systemic immunoglobulin repertoires expressed by B cells in mice in a tissueand isotype-specific manner, and provide a framework for understanding how diet and obesity could lead to pathogenic adaptive immune responses associated with IR.

\section{RESULTS}

\section{HFD selects for B cells expressing IgA with short, hydrophobic CDRH3s in multiple tissues}

As a first analysis of the IGH gene rearrangements detected in mice on HFD compared with $\mathrm{RD}$, we determined the frequencies of IGHV, IGHD, and IGHJ gene segment usage in the B cells from each tissue isolated. We considered gene segment usage separately for each antibody isotype, as there is selection for or against particular gene segments in the naive B-cell repertoire compared with memory B-cell populations. ${ }^{9,10}$ Mouse IGHV, IGHD, and IGHJ genes were aligned with the germline gene segment repertoires at the IMGT repository. ${ }^{11}$ Overall, IGHV gene segment usage was similar in HFD compared with RD mice. Supplementary Figures S1 and S2 online show the IGHV, IGHD, and IGHJ gene segment usage frequency differences in the spleen, small intestine, and VAT when five HFD mice were compared with five RD mice, plotting the top quartile of most highly rearranged IGHV gene segments, and all of the IGHD and IGHJ gene segments. When corrected for multiple hypothesis testing by the Benjamini-Hochberg procedure, no IGHV, IGHD, or IGHJ gene segment was significantly different in its frequency between the HFD and RD mice.

A key determinant of an antibody's antigen specificity is the peptide loop encoded by the CDRH3 region that spans the junctions of the joined IGHV, IGHD, and IGHJ gene segments. ${ }^{12,13}$ In humans, shorter $\mathrm{CDRH} 3$ lengths are observed in antigen-experienced B cells such as memory B cells as compared with naive $B$ cells, suggesting that there is a selection against antibodies with long $\mathrm{CDRH} 3$ sequences during antigendriven proliferation and selection. ${ }^{14,15}$ We therefore sought to determine whether there were $\mathrm{CDRH} 3$ length differences between HFD and RD mice (Figure 1a). No significant difference in $\mathrm{CDRH} 3$ length was seen for IgM in all tissues studied, after correction for multiple hypothesis testing. There was, however, a striking difference in the CDRH3 lengths of IgA in HFD compared with $\mathrm{RD}$ mice, with shorter $\mathrm{CDRH} 3$ s seen in spleen, small intestinal tissue, and VAT (Figure 1a). Interestingly, although the difference was most significant in the somatically mutated IGH rearrangements, a similar trend was seen in the germline IgA reads, suggesting that exposure to a HFD was associated with selection for shorter $\mathrm{CDRH} 3$ in both isotypeswitched unmutated B cells and those that had undergone isotype switching to IgA and somatic mutation. In contrast, IgG-expressing B cells did not show significant differences in $\mathrm{CDRH} 3$ lengths following HFD compared with $\mathrm{RD}$, suggesting that the effect of HFD is not generic for all isotype-switched B cells, but is specific to IgA-expressing B cells.

To evaluate for other systematic differences in the antibody repertoires in response to HFD, we also assessed the physicochemical properties of the $\mathrm{CDRH} 3$ amino acid sequences in each isotype. HFD was associated with an increase in hydrophobicity (shown as having higher hydropathy scores) in IgA antibodies, but not IgM or IgG antibodies (Figure 1b). The most marked increase in CDRH3 hydrophobicity was seen in VAT specimens. Thus, in the context of HFD, B cells expressing IgA with short $\mathrm{CDRH} 3 \mathrm{~s}$, with more hydrophobic amino acid residues, appear to be preferentially selected to clonally expand or persist in the tissues we examined. To investigate whether the changes to $\mathrm{CDRH} 3$ hydrophobicity were related to length, we evaluated the mean hydropathy scores as a function of CDRH3 length (Figure 2). We observed a strong negative correlation between $\mathrm{CDRH} 3$ length and hydrophobicity. This relationship was seen in both HFD and $\mathrm{RD}$ mice, and their respective linear regression lines were not significantly different in slope (Figure 2). Analysis of covariance was performed and showed CDRH3 hydropathy to be affected by CDRH3 length and diet status, and that there was significant interaction between diet status and CDRH3 length. These results indicate mice eating a HFD show increased frequencies of $\mathrm{B}$ cells expressing antibodies that are both hydrophobic and short in their CDHR3s, suggesting that selection of these $\mathrm{B}$ cells may be acting on the basis of the CDRH3 lengths, or their hydrophobicity, or both.

\section{Higher proportion of unmutated IgA in HFD}

We next systematically assessed somatic hypermutation frequencies in the IGHV gene segments associated with each antibody isotype in the HFD and RD mouse tissues. For this analysis, all of the IGH rearrangements from mice in the HFD or $\mathrm{RD}$ groups were aggregated into tissue- and isotype-specific sets, and histograms of the IGHV mutation rate distribution were generated (Figure 3a). There was a significant difference in the IgM mutation distribution between HFD and RD mice in the small intestine, expressed as the proportion of reads per mouse with $<1 \%$ mutation frequency (Figure $3 \mathbf{b}$ ). IgA in the HFD group also displayed a higher proportion of unmutated reads in the spleen and small intestine. A trend toward higher proportions of unmutated reads was also noted in IgG in the spleen and small intestine of HFD 

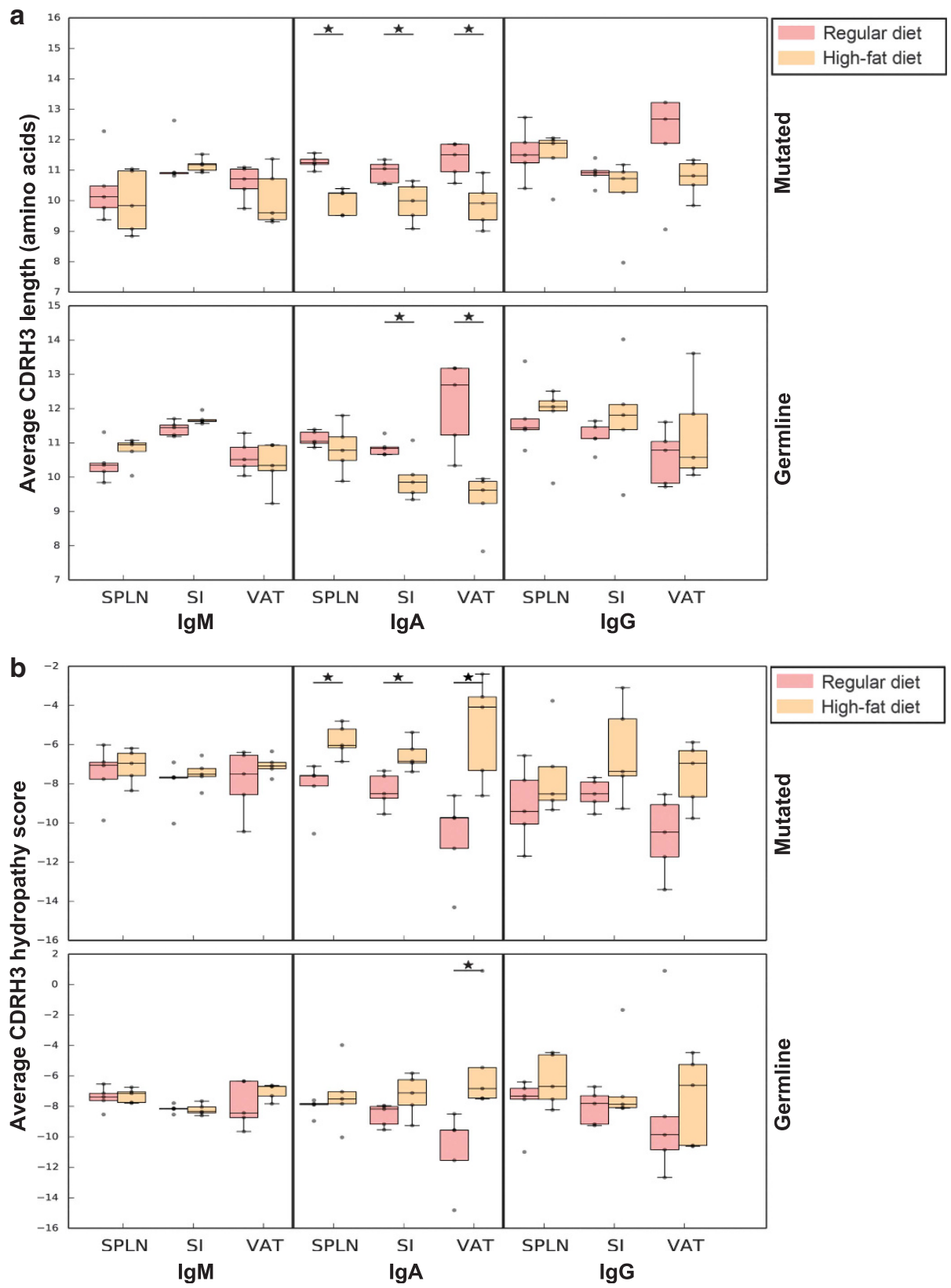

Figure 1 Complementarity-determining region-3 (CDRH3) amino acid characteristics differ between high-fat diet (HFD) and regular diet (RD) mice. $\mathrm{CDRH} 3$ length and hydropathy scores were determined and averaged per mouse for both HFD and RD mice. Results are represented in box-whisker plot, with asterisks $\left(^{*}\right)$ indicating significance after multiple hypotheses testing (MHT) correction. (a) HFD mice exhibited shorter immunoglobulin A (lgA) $\mathrm{CDRH} 3$ amino acid length. When compared with RD mice, HFD mice showed significantly shorter IgA CDRH3 amino acid length in spleen (mutated: 10.0 vs. $11.3, P<0.0004$ ), small intestine (mutated: 9.9 vs. $10.9, P<0.02$; germline: 10.0 vs. $10.9, P<0.03$ ), and visceral adipose tissue (mutated: 9.9 vs. 11.3 , $P<0.009$; germline: 9.3 vs. 12.1, $P<0.004)$. (b) HFD mice select for more hydrophobic CDRH3. When compared with RD mice, HFD mice had IgA $\mathrm{CDRH} 3$ s that were more hydrophobic (higher hydropathy score) in the small intestine (mutated: -6.6 vs. $-8.3, P<0.01$ ), visceral adipose tissue (mutated: -5.2 vs. $-10.7, P<0.008$; germline: -5.3 vs. $-10.8, P<0.03$ ), and splenic tissue (mutated: -5.8 vs. $-8.2, P<0.02$ ). SI, small intestine; SPLN, spleen; VAT, visceral adipose tissue.

mice, but these differences did not rise to significance when corrected for multiple hypothesis testing. The biological impact of elevated levels of isotype-switched but unmutated antibodies in the case of HFD mice is unknown. It is possible that these antibodies may have been stimulated by altered microbiota of mice receiving the HFD. We did not measure autoreactive or polyreactive properties of the antibodies in the HFD mice, or identify the antigens that they bind, but we note there is a precedent in the context of certain human autoimmune diseases, including lupus, for unmutated isotype-switched antibodies to be polyreactive and autoreactive. ${ }^{16-18}$ 


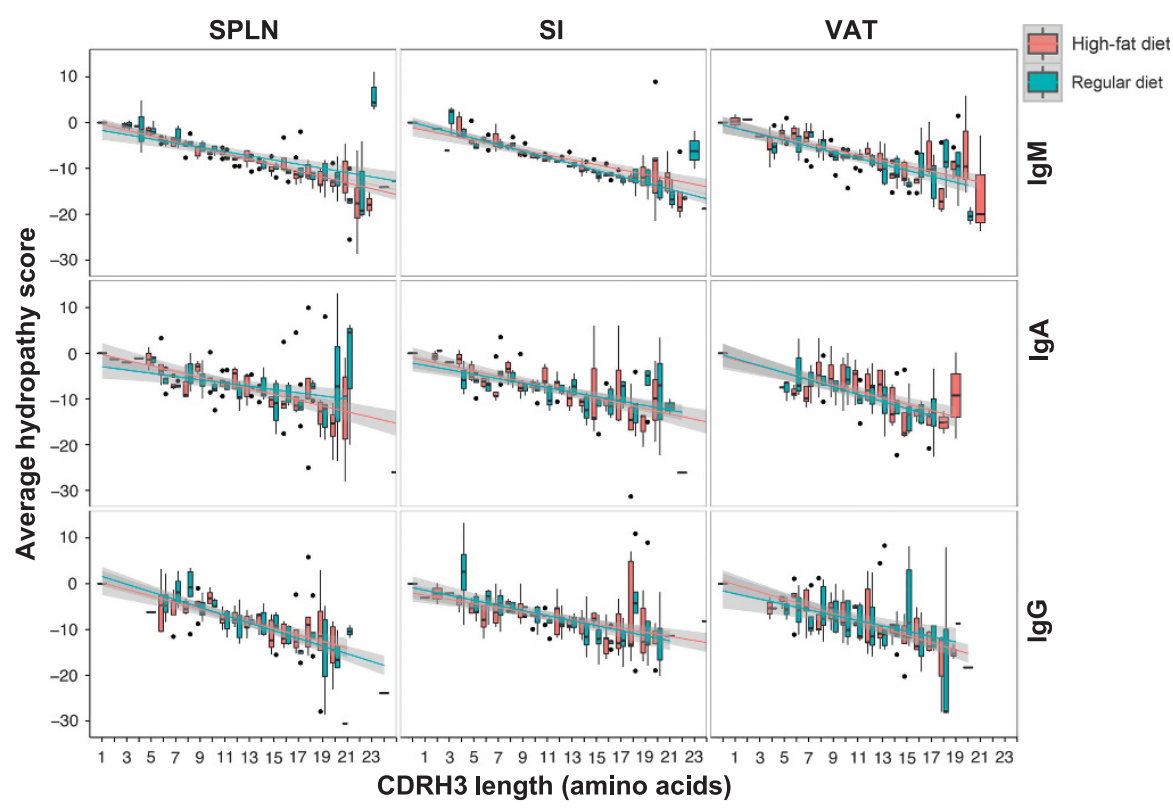

Figure 2 Complementarity-determining region-3 ( $\mathrm{CDRH} 3)$ hydropathy is negatively correlated with sequence length. $\mathrm{CDRH} 3 \mathrm{hydropathy} \mathrm{scores} \mathrm{for}$ sequences of varying length were calculated and averaged per mouse for high-fat diet (HFD) and regular diet (RD) mice. Results of each diet group are represented as a box-whisker plot per tissue and isotype studied. Regression lines are drawn for each diet group relating length to hydropathy score. There is a negative correlation between $\mathrm{CDRH} 3$ length and hydropathy score for all tissues and isotypes examined. The effect length on hydropathy is seen equally in both HFD and RD groups. SI, small intestine; SPLN, spleen; VAT, visceral adipose tissue.

\section{B cells expressing convergent antibodies are highly enriched in the VAT of mice}

"Public" or "convergent" antibody and T-cell receptor (TCR) sequences have been reported in a variety of human and mouse immunological studies, and can be the result of antigen-driven selection of similar Ig or TCR paratope sequences in different individuals, in spite of the great diversity of the B-cell and TCR repertoires. ${ }^{14,19}$ Given the prominent role of B cells in the development of IR in the HFD model, we hypothesized that there might be antigen-driven selection for convergent IGH sequences in HFD mice, and that some of these antibodies might be pathogenic. We performed a pairwise comparison of the IGH sequences derived from each mouse with the IGH sequences in all the other mice, in a tissue-specific manner, to search for similar IGH gene rearrangements shared between mice. We defined convergent sequences as those using the same IGHV and IGHJ gene segments and having identical CDRH3 length, and at least $90 \%$ similarity of CDRH3 nucleotide sequence. To normalize for the sequencing depth obtained from each mouse tissue, the number of related sequences between the two samples being compared was divided by the total number of comparisons (i.e., the product of the number of unique sequences for the two samples). A heatmap summarizing these results is presented in Figure 4a. Strikingly, we find a consistent pattern of enrichment for similar IGH sequences in the adipose tissues, particularly in mice fed the HFD. Most of these convergent rearrangements were expressed as IgM, with occasional IgG and IgA members. We next examined the somatic mutation status of the convergent adipose tissue sequences found in all mice and compared them with the convergent adipose tissue sequences found only in HFD mice but not RD mice (Figure $4 \mathbf{b}$ ). In the HFD-specific convergent rearrangements, there was an increase in the proportion of somatically mutated reads. This result is particularly interesting in light of the finding that the overall mutation rate in HFD is decreased, as shown in Figure 3b. Overall, the findings suggest that B cells expressing similar IGH rearrangements can be found in the VAT of all mice, but that there are also convergent sequences unique to the VAT of HFD mice, and these show more evidence of somatic mutation and possible affinity maturation. This could indicate the presence of an antigendriven process in response to a shared antigen or antigens in the VAT of HFD mice.

To further characterize these convergent antibodies, we clustered these convergent IGH sequences by single linkage, requiring identical IGHV and IGHJ gene usage, identical CDRH3 length, and at least $90 \%$ similarity between $\mathrm{CDRH} 3$ nucleotide sequences. The convergent rearrangements found in normal VAT repertoire grouped into 11 distinct clusters. In addition, five distinct clusters were identified in HFD VAT convergent reads. A summary of the sequence features of the convergent IGH rearrangements found in all mice, and those present specifically in the HFD mice, is presented in Table 1a, $\mathbf{b}$ and $\mathbf{c}$.

\section{DISCUSSION}

The adaptive immune system has been selected in evolution to respond sensitively to a variety of potential infectious disease threats to the host organism, but misdirected immune responses triggered by environmental exposures can also give rise to pathological consequences, as in the case of food allergies 
a $\lg M$

$\lg \mathrm{A}$

$\lg \mathbf{G}$
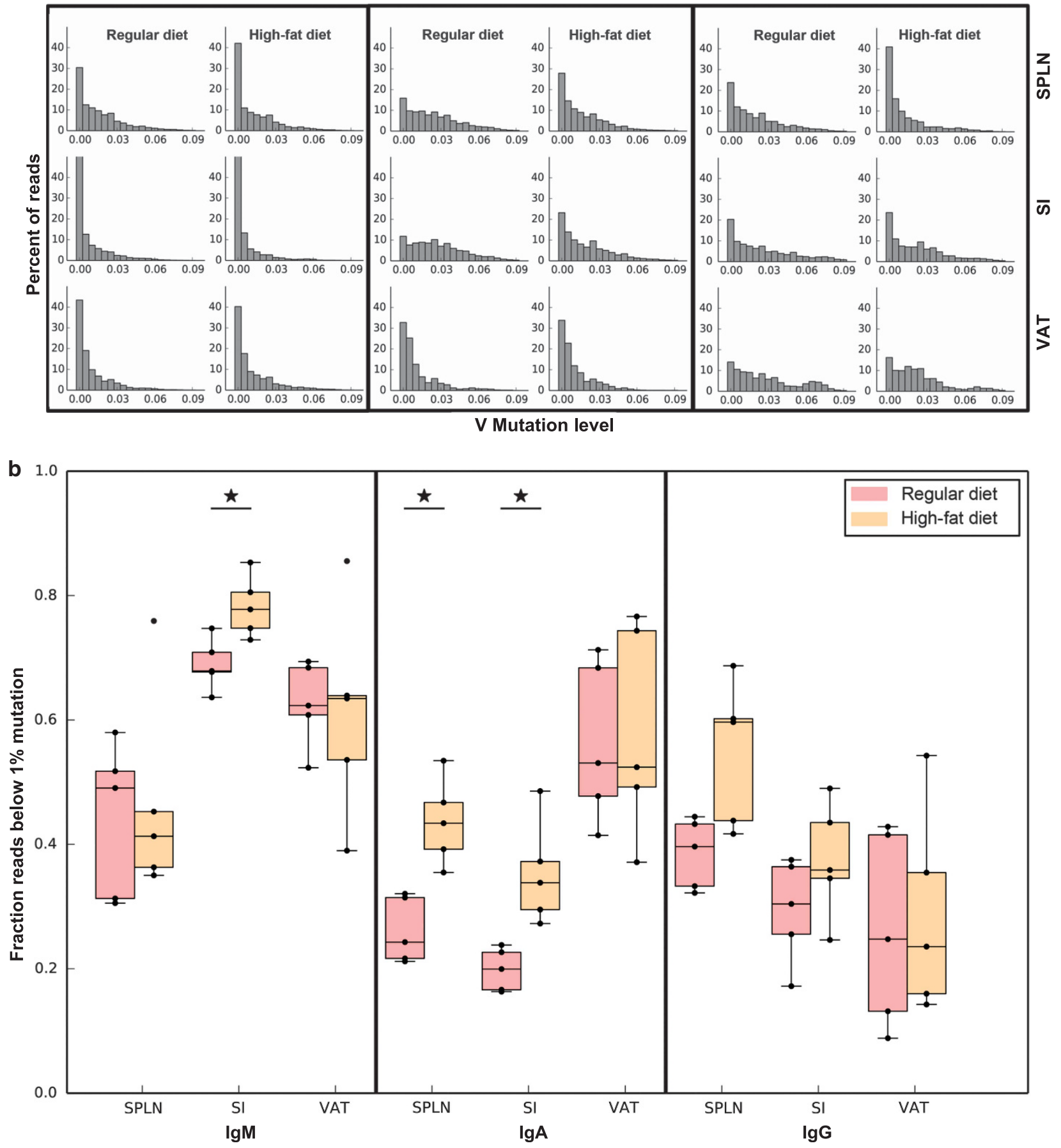

Figure 3 High-fat diet (HFD) mice have lower antibody mutation levels compared with regular diet (RD) mice. (a) All mice were grouped into either HFD or RD groups. IGHV gene mutation frequencies were calculated for each unique IGH sequence by taking the total number of mutations found in the IGHV gene segment, dividing by its full nucleotide length and expressing as a fraction (along the $\mathrm{x}$ axis) in the histogram plot. Mutation frequencies were calculated for each tissue and isotype. (b) The proportion of IGHV gene reads below a $1.0 \%$ mutation threshold was determined for each mouse on a tissue and isotype basis, and shown in the box-whisker plot. Significant comparisons after multiple hypotheses testing (MHT) correction are indicated by asterisks $\left(^{*}\right)$. HFD mice showed statistically significant higher proportion of reads below $1.0 \%$ mutation in IgA in the spleen $(0.44$ vs. $0.26, P<0.007)$ and small intestine ( 0.35 vs. $0.20, P<0.007)$, as well as IgM in the small intestine ( 0.78 vs. $0.70, P<0.02)$. SI, small intestine; SPLN, spleen; VAT, visceral adipose tissue.

and postinfectious autoimmune complications such as rheumatic heart disease, Guillain-Barre syndrome, and cold agglutinin autoimmune hemolytic anemia. It is less clear what effect the large number of nonpathogen-associated antigen exposures encountered by the host organism may have on the adaptive immune system, particularly in tissues in intimate 


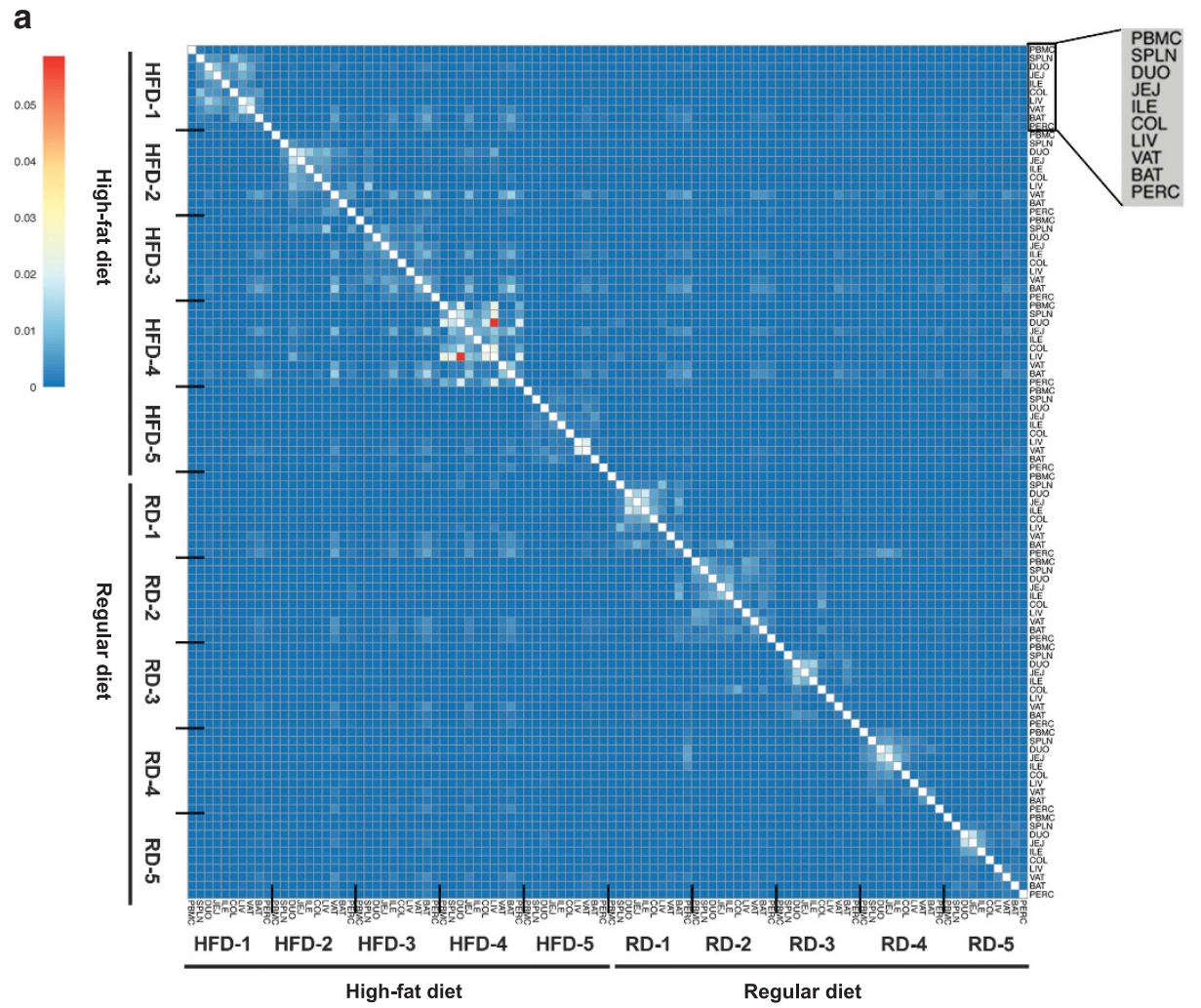

b

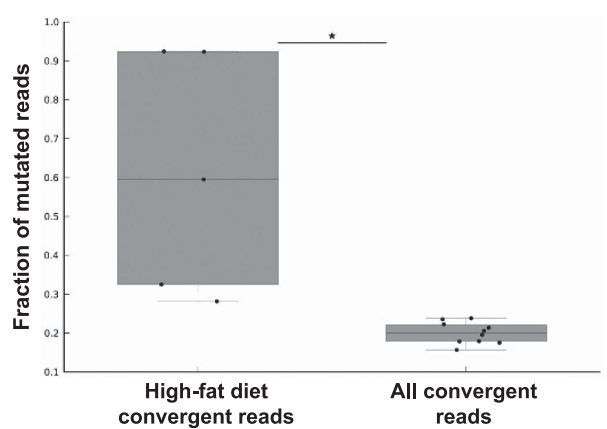

Figure 4 Convergent immunoglobulin M ( $\mathrm{IgM})$ antibody gene rearrangements in the adipose tissue of different mice. (a) A pairwise comparison of IGH reads within and between all mice and tissues was carried out to identify highly similar ("convergent") sequences shared between mice. Each individual mouse's Ig repertoire, when compared with itself, showed numerous highly similar sequences shared between different tissue compartments, as is expected from the presence of members of the same B-cell clones distributed within the mouse's body, and is seen in the large regions of lighter-colored squares along the diagonal in the heatmap. Convergent reads shared between different mice were also detected, particularly in the visceral and brown adipose tissues, with occasional overlap also seen in the small intestinal tissues, among all mice in both the high-fat diet (HFD) and regular diet (RD) groups. The proportion of convergent sequences in each comparison was corrected for sequencing read depth by dividing the total number of similar reads found between the two samples by the product of total number of reads compared in each specimen. This quotient is represented in the heatmap. Tissues are abbreviated as: BAT, brown adipose tissue; COL, colon; DUO, duodenum; ILE, ileum; JEJ, jejunum; LIV, liver; PBMC, peripheral blood mononuclear cell; PERC, peritoneal cavity cell; SPLN, spleen; VAT, visceral adipose tissue. (b) Convergent VAT reads in HFD mice have elevated proportions of mutated sequences. Mutation status of convergent visceral adipose tissue reads found in all mice (both HFD and RD mice) was compared with convergent visceral adipose tissue reads found only in HFD mice. Convergent visceral adipose tissue reads that were identified only in HFD mice showed a higher proportion of mutated reads compared with convergent visceral adipose tissue found in all mice $(0.60 \mathrm{vs}$. $0.20, P<0.002)$. ${ }^{\star} P$-value $<0.05$

contact with the environment, such as the gastrointestinal tract or lungs. Several lines of evidence indicate that obesityassociated IR induced by dietary changes may be initiated or worsened by adaptive immune responses. For example, changes to TCR repertoires can indicate clonal expansion of $\mathrm{T}$ cells that have been selected by antigenic stimuli, ${ }^{20}$ and HFD-fed mouse VAT TCRs have been reported to have a restricted repertoire compared with TCRs from lean VAT..$^{21,22}$ VAT T cells in diet induced obese ovalbumin (OVA)-specific OT-2 TCR transgenic mice develop a highly biased TCR-V $\alpha$ repertoire to non-OVA antigen(s), suggesting a non-OVA antigen-driven T-cell response. ${ }^{21}$

The HFD mouse model enables a test of the hypothesis that a simple dietary intervention that alters the composition of 
biomolecules and antigens encountered via the gastrointestinal tract can shape adaptive immune cell populations and antigen receptor repertoires in wild-type mice. We analyzed the repertoires of antibodies expressed by B cells in mice fed HFD to measure any systematic changes in the immune system under these controlled conditions. Our findings indicate that some aspects of the B-cell repertoires in mice exposed to HFD remain relatively unchanged, such as the frequency of usage of IGHV, IGHD, and IGHJ gene segments. In contrast, HFD is associated with a prominent selection for B cells expressing IgA with short CDRH3s and hydrophobic amino acid CDRH3 composition. B cells expressing IgA antibodies with short, hydrophobic CDRH3s were most prominent in the small intestine, spleen, and VAT, showing that the finding is present systemically in secondary lymphoid tissues, as well as in the gastrointestinal mucosal tissues and adipose tissues that may be most directly affected by the high-fat content of the diet. The specificity of these findings for IgA-expressing B cells, as opposed to those expressing IgM or IgG, could be consistent with a dysregulated immune reaction triggered by HFD, initiated in the gastrointestinal tract where IgA-expressing $\mathrm{B}$ cells predominate, and then extending to VAT sites and secondary lymphoid tissues.

The finding that the adaptive immune system alterations induced by HFD manifest most prominently in the IgAexpressing B cells of the gut is particularly interesting. It is possible that hydrophobic lipid-containing antigens in the gut or associated with adipose tissues lead to the selection of B cells expressing hydrophobic antibodies in these sites. Because mouse antibody $\mathrm{CDRH} 3$ length and hydrophobicity are negatively correlated, it is also possible that the HFD is leading to selection for $\mathrm{B}$ cells with shorter $\mathrm{CDRH} 3$ regions, rather than specifically acting on the hydrophobicity itself. In humans, it is well established that B cells with greater evidence of prior antigenic exposures, such as highly mutated memory B cells, show selection for shorter CDRH3 lengths, although the mechanistic reasons for this are not well understood. ${ }^{23-25}$ Another potential basis for the B-cell repertoire changes we have observed in HFD mice could be the impact of the diet on the intestinal microbiome. The intestines are the site of food absorption, and host an enormous, diverse commensal microbe population, but are also a major site of attack by pathogens. IgA, as the most abundant antibody class in the intestinal mucosal secretions, ${ }^{26}$ plays crucial roles in protecting the body from invasive pathogens and also regulating metabolism by effects on nonpathogen microbes in ways that are often intertwined. ${ }^{27}$ There is substantial evidence that obesity and type 2 diabetes are associated with an altered gut microbiome. ${ }^{28}$ Cohousing, transplantation of fecal microbiota, and antibiotic treatment have shown a causal relationship between altering gut microbiota and affecting obesity and metabolism in mouse models. ${ }^{29-31}$ Given that noninvasive commensal gut bacteria

Table 1a Nonspecific convergent antibody clones in visceral adipose tissue

\begin{tabular}{|c|c|c|c|}
\hline Representative VJCDR3 $\left(5^{\prime}-3^{\prime}\right)$ & CDR3nt length & Isotypes & Unique CDR3nt members \\
\hline IGHV1-55_IGHJ1_GCAATAGTITACTACGGTAGTAGCTACTGGTACTTCGATGTC & 42 & $\operatorname{lgG} ; \lg M ; \lg A$ & 432 \\
\hline IGHV12-3_IGHJ1_GCAGCGGACTATGATGGTTACTGGTACTTCGATGTC & 36 & $\operatorname{lgG} ; \lg M ; \lg A$ & 1,384 \\
\hline IGHV11-2_IGHJ1_ATGAGATACGGTAGTAGCTACTGGAACTTCGATGTC & 36 & IgG; IgM & 609 \\
\hline IGHV11-1_IGHJ1_ATGAGATATGGTACCTACTGGAACTTCGATGTC & 33 & $\operatorname{lgM}$ & 73 \\
\hline IGHV7-1_IGHJ1_GCAAGAGATGAATACGACTGGTACTTCGATGTC & 33 & $\lg M ; \lg A$ & 1,716 \\
\hline IGHV5-17_IGHJ1_ATGAGATATAGTAACTACTGGTACTTCGATGTC & 33 & $\lg M$ & 32 \\
\hline IGHV9-3_IGHJ1_ACAAGAGATAGTAACTGGTACTTCGATGCC & 30 & $\operatorname{lgG} ; \lg M ; \lg A$ & 507 \\
\hline IGHV1-47_IGHJ3_GCAAGGGCGGGCTITGCTTAC & 21 & lgG; IgM & 84 \\
\hline
\end{tabular}

Table 1b High-fat diet-specific convergent antibody clones in visceral adipose tissue

\begin{tabular}{|c|c|c|c|}
\hline Representative VJCDR3 $\left(5^{\prime}-3^{\prime}\right)$ & $\begin{array}{l}\text { CDR3nt } \\
\text { length }\end{array}$ & Isotypes & $\begin{array}{l}\text { Unique CDR3nt } \\
\text { members }\end{array}$ \\
\hline IGHV5-6_IGHJ1_GCAAGGCATACGTACTACGGTAGTAGCTACGGTTGGTACTTCGATGTC & 48 & $\operatorname{lgG} ; \operatorname{lgM} ; \lg A$ & 114 \\
\hline IGHV1-55_IGHJ2_GTAAGAAAAGATTACTACGGTAGTAGTTACTACTTTGACTAC & 42 & $\lg M ; \lg A$ & 25 \\
\hline IGHV11-2_IGHJ1_ATCCTCTATAGTAACTACTGGTACTITGATGTC & 33 & $\lg M$ & 5 \\
\hline IGHV4-1_IGHJ2_GCAAGAGAGACTGGGACTTCCTTTGACTAC & 30 & $\lg G ; \lg M ; \lg A$ & 104 \\
\hline IGHV1-50_IGHJ2_GCAAGAAACTACTACTTTGGCTAC & 24 & $\lg G ; \lg M ; \lg A$ & 183 \\
\hline
\end{tabular}


Table 1c High-fat diet convergent antibody clones distribution

\begin{tabular}{|c|c|c|c|c|}
\hline Representative VJCDR3 & Mouse & Tissue & Isotype & $\begin{array}{l}\text { Number of } \\
\text { unique reads }\end{array}$ \\
\hline IGHV5-6_IGHJ1_GCAAGGCATACGTACTACGGTAGTAGCTACGGTTGGTACTTCGATGTC & HFD24-1 & SPLN & $\lg M$ & 277 \\
\hline IGHV5-6_IGHJ1_GCAAGGCATACGTACTACGGTAGTAGCTACGGTTGGTACTTCGATGTC & HFD24-1 & PBMC & $\lg G$ & 244 \\
\hline IGHV5-6_IGHJ1_GCAAGGCATACGTACTACGGTAGTAGCTACGGTTGGTACTTCGATGTC & HFD24-1 & SPLN & $\lg A$ & 188 \\
\hline IGHV5-6_IGHJ1_GCAAGGCATACGTACTACGGTAGTAGCTACGGTTGGTACTTCGATGTC & HFD24-1 & LIV & $\lg G$ & 94 \\
\hline IGHV5-6_IGHJ1_GCAAGGCATACGTACTACGGTAGTAGCTACGGTTGGTACTTCGATGTC & HFD24-1 & PC & $\lg G$ & 82 \\
\hline IGHV5-6_IGHJ1_GCAAGGCATACGTACTACGGTAGTAGCTACGGTTGGTACTTCGATGTC & HFD24-1 & SPLN & $\lg G$ & 70 \\
\hline IGHV5-6_IGHJ1_GCAAGGCATACGTACTACGGTAGTAGCTACGGTTGGTACTTCGATGTC & HFD24-1 & PBMC & $\lg A$ & 19 \\
\hline IGHV5-6_IGHJ1_GCAAGGCATACGTACTACGGTAGTAGCTACGGTTGGTACTTCGATGTC & HFD24-1 & SI & $\operatorname{lgM}$ & 17 \\
\hline IGHV5-6_IGHJ1_GCAAGGCATACGTACTACGGTAGTAGCTACGGTTGGTACTTCGATGTC & HFD24-1 & VAT & $\operatorname{lgM}$ & 15 \\
\hline IGHV5-6_IGHJ1_GCAAGGCATACGTACTACGGTAGTAGCTACGGTTGGTACTTCGATGTC & HFD24-1 & PBMC & $\lg M$ & 13 \\
\hline IGHV5-6_IGHJ1_GCAAGGCATACGTACTACGGTAGTAGCTACGGTTGGTACTTCGATGTC & HFD24-1 & LIV & $\lg M$ & 11 \\
\hline IGHV5-6_IGHJ1_GCAAGGCATACGTACTACGGTAGTAGCTACGGTTGGTACTTCGATGTC & HFD24-1 & PC & $\lg M$ & 8 \\
\hline IGHV5-6_IGHJ1_GCAAGGCATACGTACTACGGTAGTAGCTACGGTTGGTACTTCGATGTC & HFD24-1 & $\mathrm{SI}$ & $\lg G$ & 4 \\
\hline IGHV5-6_IGHJ1_GCAAGGCATACGTACTACGGTAGTAGCTACGGTTGGTACTTCGATGTC & HFD24-1 & SI & $\lg A$ & 1 \\
\hline IGHV5-6_IGHJ1_GCAAGGCATACGTACTACGGTAGTAGCTACGGTTGGTACTTCGATGTC & HFD24-2 & SPLN & $\lg G$ & 2 \\
\hline IGHV5-6_IGHJ1_GCAAGGCATACGTACTACGGTAGTAGCTACGGTTGGTACTTCGATGTC & HFD24-3 & LIV & $\operatorname{lgM}$ & 4 \\
\hline IGHV5-6_IGHJ1_GCAAGGCATACGTACTACGGTAGTAGCTACGGTTGGTACTTCGATGTC & HFD24-3 & SI & $\lg G$ & 3 \\
\hline IGHV5-6_IGHJ1_GCAAGGCATACGTACTACGGTAGTAGCTACGGTTGGTACTTCGATGTC & HFD24-3 & SPLN & $\operatorname{lgM}$ & 3 \\
\hline IGHV5-6_IGHJ1_GCAAGGCATACGTACTACGGTAGTAGCTACGGTTGGTACTTCGATGTC & HFD24-3 & SI & $\lg \mathrm{M}$ & 1 \\
\hline IGHV5-6_IGHJ1_GCAAGGCATACGTACTACGGTAGTAGCTACGGTTGGTACTTCGATGTC & HFD24-3 & SPLN & $\lg A$ & 1 \\
\hline IGHV5-6_IGHJ1_GCAAGGCATACGTACTACGGTAGTAGCTACGGTTGGTACTTCGATGTC & HFD24-3 & PBMC & $\lg G$ & 1 \\
\hline IGHV5-6_IGHJ1_GCAAGGCATACGTACTACGGTAGTAGCTACGGTTGGTACTTCGATGTC & HFD24-3 & VAT & $\lg M$ & 1 \\
\hline IGHV5-6_IGHJ1_GCAAGGCATACGTACTACGGTAGTAGCTACGGTTGGTACTTCGATGTC & HFD24-4 & $\mathrm{SI}$ & $\lg G$ & 3 \\
\hline IGHV5-6_IGHJ1_GCAAGGCATACGTACTACGGTAGTAGCTACGGTTGGTACTTCGATGTC & HFD24-4 & PBMC & $\lg G$ & 2 \\
\hline IGHV5-6_IGHJ1_GCAAGGCATACGTACTACGGTAGTAGCTACGGTTGGTACTTCGATGTC & HFD24-5 & SI & $\lg G$ & 23 \\
\hline IGHV5-6_IGHJ1_GCAAGGCATACGTACTACGGTAGTAGCTACGGTTGGTACTTCGATGTC & HFD24-5 & PBMC & $\lg G$ & 22 \\
\hline IGHV5-6_IGHJ1_GCAAGGCATACGTACTACGGTAGTAGCTACGGTTGGTACTTCGATGTC & HFD24-5 & VAT & $\lg G$ & 6 \\
\hline IGHV5-6_IGHJ1_GCAAGGCATACGTACTACGGTAGTAGCTACGGTTGGTACTTCGATGTC & HFD24-5 & SPLN & $\operatorname{lgM}$ & 3 \\
\hline IGHV5-6_IGHJ1_GCAAGGCATACGTACTACGGTAGTAGCTACGGTTGGTACTTCGATGTC & HFD24-5 & $\mathrm{COL}$ & $\operatorname{lgM}$ & 3 \\
\hline IGHV5-6_IGHJ1_GCAAGGCATACGTACTACGGTAGTAGCTACGGTTGGTACTTCGATGTC & HFD24-5 & SPLN & $\lg A$ & 1 \\
\hline IGHV5-6_IGHJ1_GCAAGGCATACGTACTACGGTAGTAGCTACGGTTGGTACTTCGATGTC & HFD24-5 & PC & $\lg G$ & 1 \\
\hline IGHV5-6_IGHJ1_GCAAGGCATACGTACTACGGTAGTAGCTACGGTTGGTACTTCGATGTC & HFD24-5 & LIV & $\lg G$ & 1 \\
\hline IGHV1-55_IGHJ2_GTAAGAAAAGATTACTACGGTAGTAGTTACTACTTTGACTAC & HFD24-1 & VAT & $\lg M$ & 19 \\
\hline IGHV1-55_IGHJ2_GTAAGAAAAGATTACTACGGTAGTAGTTACTACTTTGACTAC & HFD24-1 & PC & $\lg \mathrm{M}$ & 9 \\
\hline IGHV1-55_|GHJ2_GTAAGAAAAGATTACTACGGTAGTAGTTACTACTTTGACTAC & HFD24-1 & BAT & $\lg \mathrm{M}$ & 4 \\
\hline IGHV1-55_IGHJ2_GTAAGAAAAGATTACTACGGTAGTAGTTACTACTTTGACTAC & HFD24-1 & SI & $\lg M$ & 3 \\
\hline IGHV1-55_|GHJ2_GTAAGAAAAGATTACTACGGTAGTAGTTACTACTTTGACTAC & HFD24-1 & SPLN & $\lg M$ & 2 \\
\hline IGHV1-55_IGHJ2_GTAAGAAAAGATTACTACGGTAGTAGTTACTACTTTGACTAC & HFD24-1 & PBMC & $\lg M$ & 1 \\
\hline IGHV1-55_IGHJ2_GTAAGAAAAGATTACTACGGTAGTAGTTACTACTTTGACTAC & HFD24-2 & VAT & $\operatorname{lgM}$ & 33 \\
\hline IGHV1-55_IGHJ2_GTAAGAAAAGATTACTACGGTAGTAGTTACTACTTTGACTAC & HFD24-2 & SPLN & $\operatorname{lgM}$ & 4 \\
\hline IGHV1-55_IGHJ2_GTAAGAAAAGATTACTACGGTAGTAGTTACTACTTTACTAC & HFD24-2 & PC & $\lg \mathrm{M}$ & 4 \\
\hline IGHV1-55_IGHJ2_GTAAGAAAAGATTACTACGGTAGTAGTTACTACTTTGACTAC & HFD24-2 & SI & $\lg M$ & 3 \\
\hline IGHV1-55_IGHJ2_GTAAGAAAAGATTACTACGGTAGTAGTTACTACTTTGACTAC & HFD24-2 & COL & $\lg M$ & 3 \\
\hline IGHV1-55_IGHJ2_GTAAGAAAAGATTACTACGGTAGTAGTTACTACTTTGACTAC & HFD24-2 & PBMC & $\lg M$ & 2 \\
\hline IGHV1-55_IGHJ2_GTAAGAAAAGATTACTACGGTAGTAGTTACTACTTTGACTAC & HFD24-2 & LIV & $\lg M$ & 1 \\
\hline
\end{tabular}


Table 1c (Continued)

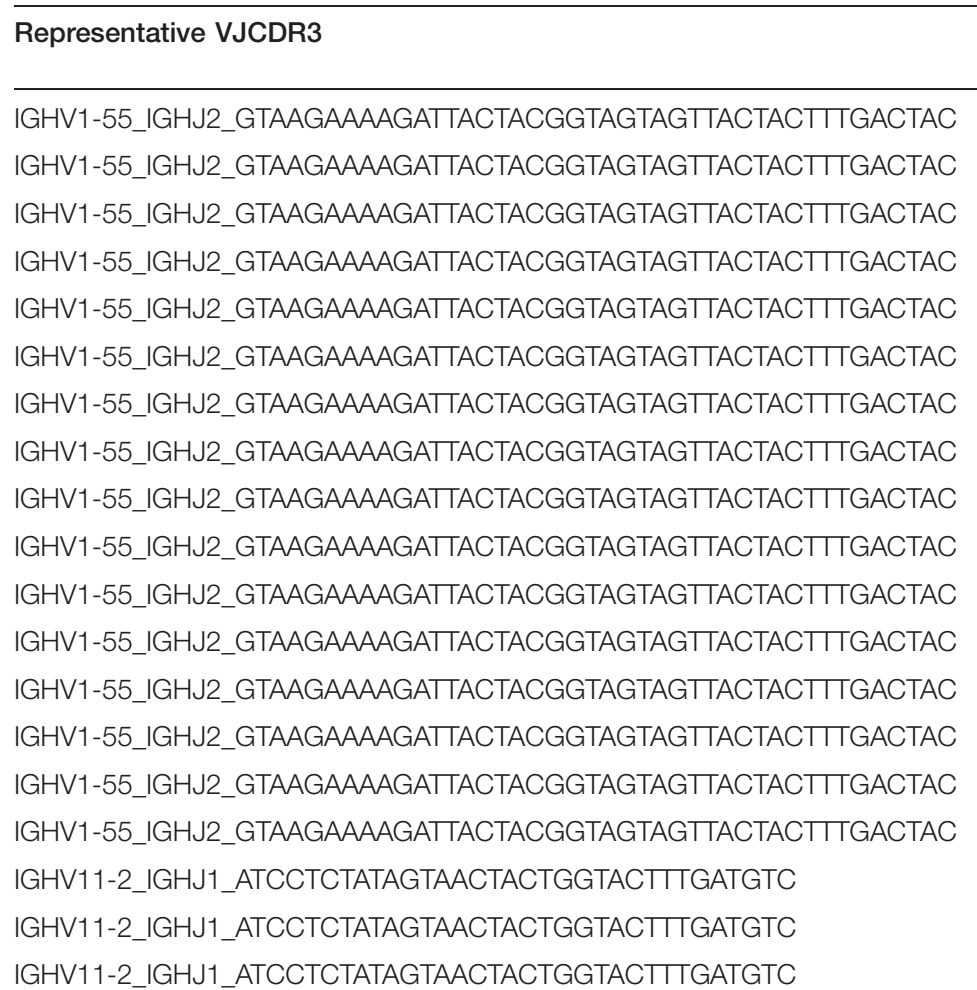
IGHV11-2_IGHJ1_ATCCTCTATAGTAACTACTGGTACTTTGATGTC IGHV11-2_IGHJ1_ATCCTCTATAGTAACTACTGGTACTTGATGTC IGHV11-2_IGHJ1_ATCCTCTATAGTAACTACTGGTACTTTGATGTC IGHV4-1_IGHJ2_GCAAGAGAGACTGGGACTTCCTITGACTAC IGHV4-1_IGHJ2_GCAAGAGAGACTGGGACTTCCTTGACTAC IGHV4-1_IGHJ2_GCAAGAGAGACTGGGACTTCCTTGACTAC IGHV4-1_IGHJ2_GCAAGAGAGACTGGGACTTCCTITGACTAC IGHV4-1_IGHJ2_GCAAGAGAGACTGGGACTTCCTITGACTAC IGHV4-1_IGHJ2_GCAAGAGAGACTGGGACTTCCTITGACTAC IGHV4-1_IGHJ2_GCAAGAGAGACTGGGACTTCCTITGACTAC IGHV4-1_IGHJ2_GCAAGAGAGACTGGGACTTCCTITGACTAC IGHV4-1_IGHJ2_GCAAGAGAGACTGGGACTTCCTITGACTAC IGHV4-1_IGHJ2_GCAAGAGAGACTGGGACTTCCTITGACTAC IGHV4-1_IGHJ2_GCAAGAGAGACTGGGACTTCCTITGACTAC IGHV4-1_IGHJ2_GCAAGAGAGACTGGGACTTCCTITGACTAC IGHV4-1_IGHJ2_GCAAGAGAGACTGGGACTTCCTITGACTAC IGHV4-1_IGHJ2_GCAAGAGAGACTGGGACTTCCTITGACTAC IGHV4-1_IGHJ2_GCAAGAGAGACTGGGACTTCCTITGACTAC IGHV4-1_IGHJ2_GCAAGAGAGACTGGGACTTCCTITGACTAC IGHV4-1_IGHJ2_GCAAGAGAGACTGGGACTTCCTITGACTAC IGHV1-50_IGHJ2_GCAAGAAACTACTACTITGGCTAC IGHV1-50_IGHJ2_GCAAGAAACTACTACTITGGCTAC IGHV1-50_IGHJ2_GCAAGAAACTACTACTITGGCTAC IGHV1-50_IGHJ2_GCAAGAAACTACTACTITGGCTAC IGHV1-50_IGHJ2_GCAAGAAACTACTACTITGGCTAC IGHV1-50_IGHJ2_GCAAGAAACTACTACTITGGCTAC IGHV1-50_IGHJ2_GCAAGAAACTACTACTITGGCTAC

\section{Mouse}

Tissue

Isotype

H

HFD24-3

VAT

HFD24-3

LIV

IgM

HFD24-3

PC

$\lg \mathrm{M}$

HFD24-3

HFD24-3

BAT

HFD24-3

SPLN

HFD24-3

COL

HFD24-4

SPLN

HFD24-4

HFD24-4

HFD24-4

HFD24-5

HFD24-5

HFD24-5

HFD24-5

HFD24-5

PC

SI

SPLN

VAT

IgM

IgM

IgM

$\lg M$

$\lg A$

$\lg M$

IgM

IgM

IgM

SI

PC

LIV

PBMC

$\lg M$

IgM

$\lg \mathrm{M}$

HFD24-1

VAT

$\lg M$

Number of unique reads

\section{$\mathrm{H}$}

HF

$$
\text { H }
$$

$$
\text { HF }
$$

\begin{tabular}{|c|c|c|c|}
\hline HFD24-3 & VAT & $\lg M$ & 1 \\
\hline HFD24-4 & SI & $\lg M$ & 1 \\
\hline HFD24-4 & VAT & $\lg M$ & 1 \\
\hline HFD24-1 & VAT & $\lg M$ & 3 \\
\hline HFD24-2 & PC & $\lg A$ & 78 \\
\hline HFD24-2 & PC & $\lg G$ & 31 \\
\hline HFD24-2 & SPLN & $\lg M$ & 4 \\
\hline HFD24-2 & PC & $\lg M$ & 1 \\
\hline HFD24-2 & PBMC & $\lg G$ & 1 \\
\hline HFD24-3 & LIV & $\lg M$ & 289 \\
\hline HFD24-3 & SPLN & $\lg M$ & 44 \\
\hline HFD24-3 & PC & $\lg A$ & 17 \\
\hline HFD24-3 & PC & $\lg M$ & 2 \\
\hline HFD24-3 & VAT & $\lg M$ & 2 \\
\hline HFD24-4 & PC & $\lg M$ & 2 \\
\hline HFD24-5 & VAT & $\lg M$ & 292 \\
\hline HFD24-5 & SPLN & $\lg M$ & 21 \\
\hline HFD24-5 & PC & $\lg G$ & 14 \\
\hline HFD24-5 & PC & $\lg M$ & 5 \\
\hline HFD24-5 & PBMC & $\lg M$ & 4 \\
\hline HFD24-1 & LIV & $\lg G$ & 44 \\
\hline HFD24-1 & PBMC & $\lg G$ & 5 \\
\hline HFD24-1 & PC & $\lg G$ & 1 \\
\hline HFD24-2 & BAT & $\lg G$ & 997 \\
\hline HFD24-2 & LIV & $\lg G$ & 551 \\
\hline HFD24-2 & VAT & $\lg G$ & 79 \\
\hline HFD24-2 & PBMC & $\lg G$ & 34 \\
\hline
\end{tabular}

$$
\text { HFD }
$$


Table 1c (Continued)

\begin{tabular}{|c|c|c|c|c|}
\hline Representative VJCDR3 & Mouse & Tissue & Isotype & $\begin{array}{l}\text { Number of } \\
\text { unique reads }\end{array}$ \\
\hline IGHV1-50_IGHJ2_GCAAGAAACTACTACTITGGCTAC & HFD24-2 & SPLN & $\lg G$ & 17 \\
\hline IGHV1-50_|GHJ2_GCAAGAAACTACTACTITGGCTAC & HFD24-2 & $\mathrm{PC}$ & $\lg G$ & 6 \\
\hline IGHV1-50_IGHJ2_GCAAGAAACTACTACTITGGCTAC & HFD24-2 & $\mathrm{COL}$ & $\lg G$ & 5 \\
\hline IGHV1-50_IGHJ2_GCAAGAAACTACTACTITGGCTAC & HFD24-3 & PBMC & $\lg G$ & 5 \\
\hline IGHV1-50_|GHJ2_GCAAGAAACTACTACTITGGCTAC & HFD24-3 & SI & $\lg G$ & 4 \\
\hline IGHV1-50_IGHJ2_GCAAGAAACTACTACTITGGCTAC & HFD24-3 & $\mathrm{PC}$ & $\lg G$ & 1 \\
\hline IGHV1-50_|GHJ2_GCAAGAAACTACTACTITGGCTAC & HFD24-3 & LIV & $\lg M$ & 1 \\
\hline IGHV1-50_IGHJ2_GCAAGAAACTACTACTITGGCTAC & HFD24-4 & SI & $\lg G$ & 6 \\
\hline IGHV1-50_IGHJ2_GCAAGAAACTACTACTITGGCTAC & HFD24-4 & COL & $\lg G$ & 5 \\
\hline IGHV1-50_IGHJ2_GCAAGAAACTACTACTITGGCTAC & HFD24-4 & SPLN & $\lg A$ & 4 \\
\hline IGHV1-50_|GHJ2_GCAAGAAACTACTACTITGGCTAC & HFD24-4 & $\mathrm{COL}$ & $\lg M$ & 1 \\
\hline IGHV1-50_IGHJ2_GCAAGAAACTACTACTITGGCTAC & HFD24-5 & LIV & $\lg G$ & 406 \\
\hline IGHV1-50_IGHJ2_GCAAGAAACTACTACTITGGCTAC & HFD24-5 & VAT & $\lg G$ & 77 \\
\hline IGHV1-50_IGHJ2_GCAAGAAACTACTACTITGGCTAC & HFD24-5 & PBMC & $\lg G$ & 34 \\
\hline IGHV1-50_IGHJ2_GCAAGAAACTACTACTITGGCTAC & HFD24-5 & $\mathrm{Sl}$ & $\lg G$ & 6 \\
\hline
\end{tabular}

Abbreviations: CDR3nt, complementarity-determining region-3 nucleotide sequence; BAT, brown adipose tissue; COL, colon; HFD, high-fat diet; Ig, immunoglobulin; LIV, liver; PBMC, peripheral blood mononuclear cell; PC, peritoneal cavity cell; SI, small intestine; SPLN, spleen; VAT, visceral adipose tissue.

can induce production of bacteria-specific IgA by mucosal B cells, ${ }^{32}$ it is possible that changes in the microbiome associated with HFD consumption can be recognized by the gutassociated B cells and alter the IgA repertoire.

We identified a set of related antibody IGH sequences that were found in the VAT of all mice analyzed in the study, both $\mathrm{HFD}$ and RD. These were predominantly expressed as IgM and can be grouped into 11 distinct clusters, each having multiple unique CDRH3 members. The presence of convergent IGH indicates that the normal B-cell repertoire in the VAT of mice has stereotypical features, similar to those of tissue-specific gamma-delta $\mathrm{T}$-cell populations previously reported in various tissue sites such as the skin, lung, or intestines in mice. ${ }^{33,34}$ Interestingly, there were five distinct sets of related IGH rearrangements that were specific to the VAT of HFD mice, and these showed a higher mutation rate than the convergent rearrangements seen in the VAT repertoires of both HFD and $\mathrm{RD}$ mice. We hypothesize that these convergent VAT B cells found exclusively in HFD mice could be involved in the development of IR, as it has been previously demonstrated that early after the initiation of HFD, B cells accumulate in the VAT, and are required for the development of IR in conjunction with $\mathrm{T}$ cells and macrophages. ${ }^{6}$ These data suggest that although there are normal resident VAT B cells with stereotyped antibody IGH sequences that constitute the normal VAT repertoire, HFD feeding may stimulate a distinct set of B cells to proliferate and undergo somatic hypermutation, potentially in response to autoantigen stimulation. It is also possible that such B cells may migrate into the VAT from elsewhere in the body in response to the HFD.

Taken together, our findings demonstrate that high-fat dietary changes lead to systemic immunoglobulin repertoire alterations, with many of these changes preferentially affecting intestinal and visceral adipose tissues, raising the possibility of a gastrointestinal-VAT immune axis shaped by the diet or other environmental antigens. The VAT in healthy mice contains a highly convergent, shared B-cell repertoire that is perturbed by increased fat consumption. These findings provide a framework for better understanding the role that $B$ cells play in diet-induced obesity and the development of insulin resistance. Although a simple dietary intervention leads to the changes observed, additional investigations are necessary to determine the mechanistic details causing the immunological disturbances. As mentioned above, immune system changes may be driven by alterations in the microbiota of the HFD mice. Furthermore, in humans, it has been shown that obesity and increased body mass index can perturb the immune system, leading to increased susceptibility to viral and bacterial infections. ${ }^{35-40}$ As HFD causes many and varied biological perturbations including obesity, contributions from other physiological changes 
associated with diet are potentially involved. Future work identifying the antigen targets of the B-cell lineages induced by HFD, and the biological effects of secreted antibodies from those cells, or alterations of the interactions of B cells with other immune cell types, should further extend these findings.

\section{METHODS}

Mice. Wild-type and HFD-fed male C57BL/6 mice were purchased from the Jackson Laboratory (Sacramento, CA) at 24 weeks of age. Animals were rested in our facility for 3 days and killed at 24 weeks of age. All mice were maintained in a specific pathogen-free, temperature-controlled environment on a $12 \mathrm{~h}$ light and dark cycle. Mice at the Jackson Laboratory were fed a diet of mouse chow for the first 6 weeks of life, then either continued with the regular mouse chow diet (RD; $15 \mathrm{kcal} \%$ fat; LabDiet, St. Louis, MO) or were switched to a HFD (60 kcal\% fat; Research Diets, New Brunswick, NJ, for 18 weeks), and were maintained on Teklad Sani-Chips bedding (Harlan, Indianapolis, IN). The Stanford University institutional animal care and use committee approved all protocols.

Tissue isolation and processing. Peripheral blood was collected via retro-orbital bleed into a potassium EDTA Vacutainer tube, transferred to a microcentrifuge tube, and snap frozen in liquid nitrogen. Peritoneal cavity cells were harvested by injecting $7 \mathrm{ml}$ of phosphatebuffered saline with $2 \mathrm{~mm}$ EDTA and $2 \%$ fetal calf serum into the peritoneal cavity. Spleen, VAT, duodenum, ileum, jejunum, colon, liver, and brown adipose tissue were harvested whole and snap frozen. Samples were stored at $-80^{\circ} \mathrm{C}$.

RNA isolation. Tissue $(75 \mathrm{mg})$ was homogenized in $750 \mu \mathrm{l}$ Trizol (Invitrogen, Carlsbad, CA) with stainless steel beads (Qiagen, Hilden, Germany) in a microtube homogenizer (Beadbug, Benchmark Scientific, Edison, NJ) for $30 \mathrm{~s}$ at $4{ }^{\circ} \mathrm{C}$. RNA was isolated as previously described. $^{7}$

IGH amplification and sequencing. IGH DNA libraries for sequencing were generated from complementary DNA prepared from total RNA isolated from mouse tissues. Complementary DNA was generated from $600 \mathrm{ng}$ of total RNA from each sample, using random hexamer primers and Superscript II (Invitrogen) reverse transcriptase. For PCR amplifications, multiplexed primers targeting the mouse framework region 1 (FR1) of the IGHV were used in combination with each isotype-specific primer. IgG isotype-specific primers were adapted from previously published primers. ${ }^{41}$ Isotype primers targeted constant region exon 1 of $\operatorname{IgA}$, IgG, or IgM. Amplification of each isotype was performed in a separate PCR reaction to avoid formation of inter-isotype chimeric PCR products. Each sample was barcoded with 8-mer primer-encoded nucleotide sequences on both the $5^{\prime}$ and $3^{\prime}$ ends of the amplicons. A list of primers used is shown (Supplementary Table S1). PCR amplification was performed in two steps. In the first step, amplicons were generated using primers with the partial Illumina (San Diego, CA) adapter, barcode and locus-specific sequence. A final $30 \mu \mathrm{l}$ volume was used in the first PCR amplification. A second PCR was then performed to complete the Illumina adapter sequence and to ensure that final PCR product was not amplified to saturation; we used $1 \mu \mathrm{l}$ from the first PCR product with a final $20 \mu \mathrm{l}$ volume. The conditions for the first step PCR were 35 cycles at: melt $94^{\circ} \mathrm{C}$ for $30 \mathrm{~s}$, anneal at $56^{\circ} \mathrm{C}$ for $45 \mathrm{~s}$, and extend at $72^{\circ} \mathrm{C}$ for $90 \mathrm{~s}$. Conditions of the second PCR step were 12 cycles at: melt $94{ }^{\circ} \mathrm{C}$ for $30 \mathrm{~s}$, anneal at $60{ }^{\circ} \mathrm{C}$ for $45 \mathrm{~s}$, and extend at $72^{\circ} \mathrm{C}$ for $90 \mathrm{~s}$. Amplified product size was verified by electrophoresis with a $1.5 \%$ agarose gel. Products were then pooled, purified by agarose gel electrophoresis and extraction, and sequenced with an Illumina MiSeq instrument.

Analysis of IGH sequence data. Paired-end sequences were merged, demultiplexed based on exact matching to primer sequences and primer-encoded barcode sequences to determine sample assignment, and primer-trimmed. Next, the variable (IGHV), diversity (IGHD), and joining (IGHJ) gene segment usage in each rearrangement, as well as mutations from germline, was identified using the IgBlast program. ${ }^{8}$ Isotype identity was established by exact match to non-primerencoded sequence within exon 1 of the sequenced constant region. The number of reads and clones for each mouse, tissue type, and isotype are presented in Supplementary Tables S2 and S3. CDRH3 was determined as the sequence flanked on the $5^{\prime}$ end by the end of FR3 as identified by IgBlast, and on the $3^{\prime}$ by FR 4 boundary determined by position weight matrix search trained to identify the WGQG motif and sequence variants. To avoid overinterpretation of sequencing errors as somatic mutations in IGH gene rearrangements, a threshold of $1 \%$ mutation in the IGHV gene segment was used to distinguish mutated sequences from unmutated sequences. Exactly matching IGH nucleotide sequences from the same sample were collapsed into a single read for further analysis.

SUPPLEMENTARY MATERIAL is linked to the online version of the paper at http://www.nature.com/mi

\section{ACKNOWLEDGMENTS}

The project was supported by the NIH NIAID grant R01DK096038 (to E.G.E., principal investigator).

\section{AUTHOR CONTRIBUTIONS}

T.D.P.: designed and performed experiments, data analysis, results interpretation, and drafted and revised manuscript; M.H.Y.C.: designed and performed experiments, data analysis, and revised manuscript; K.M.R. and K.J.L.J.: data analysis, results interpretation, and revised manuscript; K.D.N. and J.-Y.L.: performed experiments, acquired data, and revised manuscript; J.G.: results interpretation and revised manuscript;: E.G.E and S.D.B.: designed experiments, results interpretation, and revised manuscript.

\section{DISCLOSURE}

The authors declared no conflict of interest.

(c) 2017 Society for Mucosal Immunology

\section{REFERENCES}

1. Chatzigeorgiou, A. \& Chavakis, T. Immune cells and metabolism. Handb. Exp. Pharmacol. 233, 221-249 (2015).

2. Petersen, K.F., Dufour, S., Befroy, D., Garcia, R. \& Shulman, G.I. Impaired mitochondrial activity in the insulin-resistant offspring of patients with type 2 diabetes. N. Engl. J. Med. 350, 664-671 (2004).

3. Kadowaki, T., Yamauchi, T., Kubota, N., Hara, K., Ueki, K. \& Tobe, K. Adiponectin and adiponectin receptors in insulin resistance, diabetes, and the metabolic syndrome. J. Clin. Invest. 116, 1784-1792 (2006).

4. Morioka, T. et al. Disruption of leptin receptor expression in the pancreas directly affects beta cell growth and function in mice. J. Clin. Invest. 117, 2860-2868 (2007).

5. Niswender, K.D. \& Magnuson, M.A. Obesity and the beta cell: lessons from leptin. J. Clin. Invest. 117, 2753-2756 (2007).

6. Winer, D.A. et al. B cells promote insulin resistance through modulation of T cells and production of pathogenic IgG antibodies. Nat. Med. 17, 610-617 (2011).

7. Untergasser, A. RNAprep - Trizol combined with Columns (2008). Available at http://www.untergasser.de/lab/protocols/rna_prep_comb_trizol_v1_ O.htm.

8. Ye, J., Ma, N., Madden, T.L. \& Ostell, J.M. IgBLAST: an immunoglobulin variable domain sequence analysis tool. Nucleic Acids Res. 41, W34-W40 (2013).

9. Yancopoulos, G.D., Malynn, B.A. \& Alt, F.W. Developmentally regulated and strain-specific expression of murine VH gene families. J. Exp. Med. 168, 417-435 (1988).

10. Wesemann, D.R. et al. Microbial colonization influences early B-lineage development in the gut lamina propria. Nature 501, 112-115 (2013). 
11. Lefranc, M.-P. IMGT-ONTOLOGY and IMGT databases, tools and web resources for immunogenetics and immunoinformatics. Mol. Immunol. 40, 647-659 (2004).

12. Jung, D., Giallourakis, C., Mostoslavsky, R. \& Alt, F.W. Mechanism and control of $\mathrm{V}(\mathrm{D}) \mathrm{J}$ recombination at the immunoglobulin heavy chain locus. Annu. Rev. Immunol. 24, 541-570 (2006).

13. Xu, J.L. \& Davis, M.M. Diversity in the CDR3 region of $\mathrm{V}(\mathrm{H})$ is sufficient for most antibody specificities. Immunity 13, 37-45 (2000).

14. Jackson, K.J. et al. Human responses to influenza vaccination show seroconversion signatures and convergent antibody rearrangements. Cell Host Microbe 16, 105-114 (2014).

15. Tian, C., Luskin, G.K., Dischert, K.M., Higginbotham, J.N., Shepherd, B.E. \& Crowe, J.E. Jr. Immunodominance of the VH1-46 antibody gene segment in the primary repertoire of human rotavirus-specific B cells is reduced in the memory compartment through somatic mutation of nondominant clones. J. Immunol. 180, 3279-3288 (2008).

16. Zhang, J., Jacobi, A.M., Wang, T., Berlin, R., Volpe, B.T. \& Diamond, B. Polyreactive autoantibodies in systemic lupus erythematosus have pathogenic potential. J. Autoimmun. 33, 270-274 (2009).

17. Meffre, E. \& Wardemann, H. B-cell tolerance checkpoints in health and autoimmunity. Curr. Opin. Immunol. 20, 632-638 (2008).

18. Samuels, J., Ng, Y.S., Coupillaud, C., Paget, D. \& Meffre, E. Impaired early B cell tolerance in patients with rheumatoid arthritis. J. Exp. Med. 201, 1659-1667 (2005).

19. Parameswaran, P. et al. Convergent antibody signatures in human dengue. Cell Host Microbe 13, 691-700 (2013).

20. Newell, E.W. \& Davis, M.M. Beyond model antigens: high-dimensional methods for the analysis of antigen-specific T cells. Nat. Biotechnol. 32 , 149-157 (2014).

21. Winer, $\mathrm{S}$. et al. Normalization of obesity-associated insulin resistance through immunotherapy. Nat. Med. 15, 921-929 (2009).

22. Yang, $\mathrm{H}$. et al. Obesity increases the production of proinflammatory mediators from adipose tissue T cells and compromises TCR repertoire diversity: implications for systemic inflammation and insulin resistance. J. Immunol. 185, 1836-1845 (2010).

23. Wang, C. et al. Effects of aging, cytomegalovirus infection, and EBV infection on human B cell repertoires. J. Immunol. 192, 603-611 (2014).

24. Rosner, K., Winter, D.B., Tarone, R.E., Skovgaard, G.L., Bohr, V.A. \& Gearhart, P.J. Third complementarity-determining region of mutated $\mathrm{VH}$ immunoglobulin genes contains shorter $\mathrm{V}, \mathrm{D}, \mathrm{J}, \mathrm{P}$, and $\mathrm{N}$ components than non-mutated genes. Immunology 103, 179-187 (2001).

25. McHeyzer-Williams, M.G., McLean, M.J., Lalor, P.A. \& Nossal, G.J. Antigen-driven B cell differentiation in vivo. J. Exp. Med. 178, 295-307 (1993).
26. Pabst, R., Russell, M.W. \& Brandtzaeg, P. Tissue distribution of lymphocytes and plasma cells and the role of the gut. Trends Immunol. 29, 206-208. author reply 9-10 (2008).

27. Shulzhenko, N. et al. Crosstalk between B lymphocytes, microbiota and the intestinal epithelium governs immunity versus metabolism in the gut. Nat. Med. 17, 1585-1593 (2011).

28. Karlsson, F.H. et al. Gut metagenome in European women with normal, impaired and diabetic glucose control. Nature 498, 99-103 (2013).

29. Upadhyay, V. etal. Lymphotoxin regulates commensal responses to enable diet-induced obesity. Nat. Immunol. 13, 947-953 (2012).

30. Ridaura, V.K. et al. Gut microbiota from twins discordant for obesity modulate metabolism in mice. Science 341, 1241214 (2013).

31. Cox, L.M. et al. Altering the intestinal microbiota during a critical developmental window has lasting metabolic consequences. Cell 158, 705-721 (2014).

32. Hapfelmeier, S. et al. Reversible microbial colonization of germ-free mice reveals the dynamics of IgA immune responses. Science 328, 1705-1709 (2010).

33. Allison, J.P. \& Havran, W.L. The immunobiology of $T$ cells with invariant gamma delta antigen receptors. Annu. Rev. Immunol. 9, 679-705 (1991).

34. Ramirez, K., Witherden, D.A. \& Havran, W.L. All hands on DE(T)C: epithelial-resident gammadelta $T$ cells respond to tissue injury. Cell. Immunol. 296, 57-61 (2015).

35. Karlsson, E.A. \& Beck, M.A. The burden of obesity on infectious disease. Exp. Biol. Med. 235, 1412-1424 (2010).

36. Sheridan, P.A. et al. Obesity is associated with impaired immune response to influenza vaccination in humans. Int. J. Obes. 36, 1072-1077 (2012).

37. Shaikh, S.R. et al. The effects of diet-induced obesity on B cell function. Clin. Exp. Immunol. 179, 90-99 (2015).

38. Langley, G. et al. The impact of obesity and diabetes on the risk of disease and death due to invasive group A Streptococcus infections in adults. Clin. Infect. Dis. 62, 845-852 (2016).

39. Lizza, B.D. etal. Impact of body mass index on clinical outcomes in patients with gram-negative bacteria bloodstream infections. J. Infect. Chemother. 22, 671-676 (2016).

40. Sun, Y. et al. Weight and prognosis for influenza A(H1N1)pdm09 infection during the pandemic period between 2009 and 2011: a systematic review of observational studies with meta-analysis. Infect. Dis. 48, 813-822 (2016).

41. Tiller, T., Busse, C.E. \& Wardemann, H. Cloning and expression of murine lg genes from single B cells. J. Immunol. Methods 350, 183-193 (2009). 